\title{
Metagenomic Analysis Reveals the Microbiome and Antibiotic Resistance Genes in Guangdong Yellow- feathered Indigenous Chicken
}

\section{Yibin Xu}

South China Agricultural University https://orcid.org/0000-0001-5280-4324

Guo Lijin

South China Agricultural University

Yulin Huang

South China Agricultural University

Siyu Zhang

South China Agricultural University

Xiquan Zhang

South China Agricultural University

Qinghua Nie ( $\nabla$ nqinghua@scau.edu.cn )

South China Agricultural University https://orcid.org/0000-0003-3934-3820

\section{Research}

Keywords: yellow-feathered broiler, metagenomics, microbiome, antibiotic resistant gene

Posted Date: October 12th, 2021

DOI: https://doi.org/10.21203/rs.3.rs-954858/v1

License: () (1) This work is licensed under a Creative Commons Attribution 4.0 International License. Read Full License 


\section{Abstract}

Background: The yellow-feathered broiler has great nutritional value and a huge industrial scale as a traditional broiler in China. The intestinal microbes have been shown to correlate with poult performance, and serve as an essential reservoir of antibiotic resistance gene (ARG), and antimicrobial resistance is a major public health concern. We investigated the functional characteristics of the six different breeds of yellow-feathered broiler's (Huiyang Bearded chicken, Xinghua chicken, Huaixiang chicken, Zhongshan Shalan chicken, Qingyuan Patridge chicken, Yangshan chicken) gut microbiome through metagenomics sequencing, to investigate the functions and ARGs in the intestinal microbial communities.

Results: The results found that microbial populations of yellow-feathered broilers were mainly dominated by Bacteroidetes and Firmicutes at phylum level and Bacteroides at genus level, and the qingyuanPatridge chicken had a significantly higher level of Prevotella genus than the other five breeds of chicken. PCoA analysis indicated significant differences in the structures of the microbial communities and ARGs based on binary-jaccard distance (analysis of similarities ANOSIM $R=0.747, P=0.001$ and $R=0.525, P=0.001$, respectively) among the six breeds of chicken. Analyses using KEGG, eggNOG, and CAZy databases indicated that the pathways of metabolism, genetic information processing and glucoside hydrolase were significantly enriched in the yellow-feathered broilers. We also identified 989 ARGs and mainly composed of ARGs that confer tetracycline, multidrug and aminoglycoside represented more than $80 \%$ of the fecal resistomes, and then the $\operatorname{tet}(Q)$ gene is the most abundant in yellow-feathered chickens. In addition, we found a greater abundance of resistance genes detected in the Xinghua chicken.

Conclusions: Our study presents a metagenomic resource of indigenous chicken cecum microbiomes from six breeds of yellow-feathered broiler. It demonstrates the measure of metagenomic method for annotating microbial taxonomy and functional abundance. With the help of metagenomic, we have shown the difference in the gut microbial community structure of indigenous broiler breeds, and their potential to ARGs and antibiotic resistance. Xinghua chicken has great resistance to antibiotics. Overall, the current data provides a significant improvement to our knowledge and study of the gut microbiome and antibiotic resistance, especially in the indigenous yellow-feathered Chinese chicken.

\section{Background}

In the past several decade years, the broiler industry has developed rapidly in China and its production has ranked the second largest in the world after the USA. In the broiler industry, the yellow-feathered chickens and white-feathered chickens are mainly bred. The yellow-feathered chickens contribute around $50 \%$ of the chicken meat produced in China, with annual production exceeding four billion birds [1]. The yellow-feathered broiler production is concentrated in the south area of China, led by Guangdong Province. The yellow-feathered chickens, otherwise known as three-yellow chickens because of their characteristic yellow beak, feathers, and feet [2]. Their importance is evidenced by the incredible leap in their demand. For instance, the production of yellow-feathered chicken meat in China reached 4,445 kt in 2015 , representing $31.8 \%$ of the national broiler meat yields [3]. Among the various indigenous chicken 
breeds in Guangdong, 6 indigenous chicken breeds has been preserved due to their distinctive appearance and varied genetic resources diversities, including Huiyang Bearded chicken, Xinghua chicken, Huaixiang chicken, Zhongshan Shalan chicken, Qingyuan Patridge chicken, Yangshan chicken [4]. The 6 varieties may have different genetic backgrounds.

The gut microbial genome is considered as the host's second genome, which is closely related to the DNA genome [5], and the intestinal microbiome population plays an important role in their metabolism,immunity and host physiology $[6,7]$. Metagenomics sequencing has become a powerful method for analyzing microbial communities. Compared with 16 s sequencing, metagenomics can output higher resolution assemblies. Based on metagenomics application, less abundant taxa and their functional potential could be identified, which could show more microbial diversity within and between samples. Intestinal microbiota is considered a crucial organ that plays an integral role in maintaining the health of the host by modulating several physiological functions including nutrition, metabolism, and immunity $[8,9]$. The digestive process is strongly linked to gut microbiota; nutrient absorption, feed digestibility, energy harvest and therefore productivity are influenced by microbiota composition and diversity $[10,11]$. The gut microbial genomes of the 6 varieties above are still not clear and need further characterization. Meanwhile, intestinal microbiomes have been investigated to understand the prevalence of ARGs as a vast reservoir. With the rapid decline in production costs and the increased use of antibiotics, more and more sub-therapeutic doses of antibiotics are used in the breeding industry to promote growth and prevent diseases, and that posing high pressure on Chinese poultry produce. It is estimated that the global consumption of antimicrobials used for chickens, pigs, and cattle will increase by $67 \%$, from 63,151 tons in 2010 to 105,596 tons in 2030 [12]. Improper use of antibiotics in many environments leads to the spread of ARGs in China [13]. Frequent use of antibiotics in livestock animals and humans results in the propagation of ARGs and is becoming a major global health issue.

In this study, we investigated the cecum microbiota using whole metagenome sequencing, to accomplish the following three main goals: (1) investigate the taxonomical abundance among different breeds of broiler through assembly and alignment; (2) functionally annotate the microbes through KEGG, eggNOG, and CAZy analyses and look for functional abundance, and finally (3) identify antibiotic resistant genes in the cecum microbiota. This data improves our understanding of the chicken cecum microbiome and it is a valuable resource for studying antibiotic resistance in indigenous Chinese chickens.

\section{Methods}

\section{Experimental animal}

We collected cecal contents samples from a total of 24 representative broilers from 6 indigenous breeds of commercial chicken farms in Guangdong Province of China (Huiyang Bearded chicken: HZHXC, Xinghua chicken: FKXHC, Huaixiang chicken: XYHXC, ZhongshanShanlan chicken: ZSSLC, QingyuanPatridge chicken: QYMC, Yangshan chicken: YSC). Broilers were killed by cervical dislocation 
and the abdomen was opened. Cecum was excised and content was collected and preserved in the sterile containers, and finally stored at $-80^{\circ} \mathrm{C}$ until DNA extraction.

\section{DNA extraction, library construction, and sequencing}

DNA from each sample was extracted using a PowerSoil@ DNA Isolation Kit following the manufacturer's instructions. The DNA concentration and purity were measured using Nanodrop 2000 and agarose gel electrophoresis, respectively. Used Bioruptor Pico Sonication System (ultrasonication) to fragment the qualified DNA samples, and then use amplicon sequencing for library construction. The constructed library was first performed examine of library quality, and the Illumina sequencing platform was used for metagenomic sequencing of the qualifying library. Experiments were conducted at Biomarker Technologies Co., Ltd. (Beijing, China).

\section{Gene prediction and taxonomy annotation}

Used Trimmomatic (version 0.33) and bowtie2 (version 2.2.4) software to perform quality control and host filtering on the raw reads obtained by sequencing. After obtaining clean reads, used software MEGAHIT (Version 1.1.2) to filter contig sequences shorter than $300 \mathrm{bp}$ for metagenomic assembly, and gene prediction was done by MetaGeneMark (Version 3.26). The information of the gene set for each sample was obtained through CD-HIT (Version 4.6.6) by keeping clustering threshold at $95 \%$ and coverage threshold at $90 \%$. For taxonomy annotation, metagenomic reads from each sample were compared against NR database of NCBI by using Diamond to annotate each metagenomic homolog.

\section{Functional annotation}

KEGG databases included a large of important pathway information about the biological system, and providing a way to understand the microbial functions in hosts from metagenomic datasets. EggNOG database was regarded as a significant public resource for functional annotation that based on Orthologous Groups (OGs) of proteins at different taxonomic levels. Here, we mapped the amino acid sequences of gene catalog into the proteins in eggNOG and KEGG databases using DIAMOND program

with default parameters (e value $\leq 1 \mathrm{e}^{-5}$ ), then selected the highest-scoring annotated hit. In addition, we used hmmer software (version 3.0) to compare the protein sequence of non-redundant genes with CAZy database (version 6.0) to find the carbohydrate active enzymes in the genome. We compared the relative abundance of genes at same functional level with relative abundance of each functional category. The non-redundant yellow-feathers broilers gut microbiome gene set was further searched against the Comprehensive Antibiotic Resistance Databases (CARD) to assess the presence of antibiotic resistance genes through using RGI software (version 4.2.2).

\section{Statistical analysis}

Statistical comparisons were done using one-way analysis of variation tests, and visualized data were obtained with Graphpad prism 8.0 software. A $P$ value of $<0.05$ was regarded as statistically significant. 
The a-diversity and $\beta$-diversity on the genus, KO, COG and CAZy profile was calculated in each sample and visualized data analysis were was performed using BMKCloud (www.biocloud.net).

\section{Result}

\section{Metagenomic sequencing and gene prediction}

Metagenomic sequencing generated 291 billion raw reads corresponding to $123 \mathrm{Gbp}$ of raw data from the cecum content of the 24 broilers. Perform low-quality read filtering on each sample data to obtain clean reads (Supplementary Table S1). After assembling and predict sequencing data, display an information about reads, length of open reading frame (ORF), N50 etc. of each broiler is shown in Table 1, and the gene length distribution of the non-redundant gene set is shown in histogram (Fig. 1a). We identified 7.11 million non-redundant genes, with an average ORF length for $551 \mathrm{bp}$. Moreover, we investigated the difference in gene number among six group, and found that the number of nonredundant genes in higher in FKXHC compared to other five groups, but the difference is not significant as shown in the scatter plot (Fig. 1b). The numbers of common and unique genes among the six groups are shown in (Fig. 1C), and 198778 genes were found to be common in both regions.

Table 1. Sequenced reads analysis and assembly statistics 


\begin{tabular}{|llllll|}
\hline Sample & Contig Num. & Total Len. (bp) & N50 (bp) & GC (\%) & Mapped (\%) \\
\hline FKXHC1 & 392222 & 555133757 & 1777 & 48.05 & 94.65 \\
\hline FKXHC2 & 433139 & 628311260 & 1884 & 49.23 & 93.86 \\
\hline FKXHC3 & 262011 & 458872158 & 2874 & 51.35 & 95.88 \\
\hline FKXHC4 & 407587 & 608707582 & 2009 & 48.44 & 94.35 \\
\hline HZHXC1 & 237593 & 361955020 & 2072 & 48.56 & 88.32 \\
\hline HZHXC2 & 296745 & 484357564 & 2374 & 48.34 & 95.15 \\
\hline HZHXC3 & 308913 & 464894041 & 2005 & 48.71 & 93.88 \\
\hline HZHXC4 & 295257 & 476343903 & 2362 & 51.55 & 94.65 \\
\hline QYMC1 & 219833 & 401060089 & 3327 & 48.47 & 94.28 \\
\hline QYMC2 & 239798 & 355326828 & 1943 & 48.01 & 88.95 \\
\hline QYMC3 & 321474 & 525704855 & 2419 & 48.74 & 94.64 \\
\hline QYMC4 & 263335 & 426235271 & 2359 & 50.54 & 93.82 \\
\hline XYHXC1 & 394324 & 549891671 & 1734 & 47.23 & 92.43 \\
\hline XYHXC2 & 379211 & 540337865 & 1832 & 48.05 & 94.05 \\
\hline XYHXC3 & 401311 & 571101588 & 1812 & 50.01 & 92.6 \\
\hline XYHXC4 & 385463 & 546185646 & 1823 & 48.97 & 92.38 \\
\hline YSC1 & 237314 & 226163115 & 893 & 46.98 & 77.19 \\
\hline YSC2 & 375498 & 508359646 & 1646 & 49.05 & 91.58 \\
\hline YSC3 & 338497 & 498029370 & 1936 & 47.65 & 93.23 \\
\hline YSC4 & 370584 & 528209061 & 1811 & 47.8 & 91.82 \\
\hline ZSSLC1 & 282670 & 442677074 & 2215 & 48.64 & 94.43 \\
\hline ZSSLC2 & 348071 & 512786581 & 1914 & 48.06 & 95.04 \\
\hline ZSSLC3 & 315508 & 464274920 & 1903 & 48.85 & 94.97 \\
\hline ZSSLC4 & 348665 & 518056690 & 1978 & 47.58 & 95.03 \\
\hline
\end{tabular}

\section{Microbial Taxonomy Annotation}

The scaftigs were aligned against the non-redundant protein (NR) database to get the microbial population composition and relative abundance information. Phylum and genus level distributions for 
individual samples are shown in Fig. 2a and b. At the phylum level, Bacteroidetes and Firmicutes were two dominant phyla in all samples, accounting for $75-98 \%$ of the total bacterial community. At the genus level, 3072 genera were identified across all group (Supplementary Table 2). The Bacteroides and Prevotella were dominant genus in QYMC group (13.9\% and $15.5 \%$, respectively), and the dominant genus of the other five groups is only Bacteroides. Interestingly, we compared the abundance of the top 30 abundance genus in the six groups, and found that only the QYMC group had a significantly higher level of Prevotella than the other five groups (Fig. $2 \mathrm{~d}$ and e). To distinguish between the variations of host gut microbiomes, the a-diversity and $\beta$-diversity of microbial communities among different chicken groups were also evaluated. The chao1 index (the abundance within samples) demonstrated the number of genera in XYHXC group was higher than in other groups, whereas the difference is not obvious. The shannon index (the diversity within samples) of the cecum microflora was more diverse in HZHXC and ZSSLC group (Supplementary Figure S1). In addition, the results of Principle Coordination Analysis (PCoA) and Non-Metric Multi-Dimensional Scaling analysis (NMDS) for dimension reduction analysis based on the binary_jaccard distance revealed the dissimilarity of bacterial communities among all groups (Fig. $3 a$ and b). The closer the sample points on the scatter chart, the greater the similarity of the composition. Analysis of similarity (ANOSIM) is a nonparametric test that assesses whether variation between groups is significantly greater than variation within groups, which helps to evaluate the reasonability of the division of groups $(\mathrm{R}=0.747, P=0.001)$ (Fig. 3c).

\section{Functional annotation}

We then predicted the functional and metabolic pathways of the broilers' gut microbiome non-redundant (NR) genes sequenced based on the following functional databases: Kyoto Encyclopedia of Genes and Genomes (KEGG), Evolutionary genealogy of genes, Non-supervised Orthologous Groups (eggNOG), and Carbohydrate-Active enzymes Database (CAZy). From KEGG pathway results, we found that a large number of pathways belonged to metabolism groups (77.0\%), including carbohydrate metabolism (10.3\%), Amino acid metabolism (8.6\%), nucleotide metabolism (6.2\%), metabolism of cofactors and vitamins (6.1\%), energy metabolism (4.4\%), glycan biosynthesis and metabolism (2.7\%), and lipid metabolism (2.4\%) (Fig. 4a). The eggNOG analysis showed most of the gene functions remained unclear $(18 \%)$, while the known functions are relatively more abundant in replication, recombination and repair (6.7\%); translation, ribosomal structure and biogenesis (5.3\%); cell wall (4.8\%) and amino acid transport and metabolism (4.6\%) (Fig. 4b). Meanwhile, the CAZy database analysis exposed that the most enzymes are classified as glycoside hydrolases $(44.9 \%)$, followed by classified by glycosyltransferases (22.5\%), polysaccharide lyases (13.5\%) and carbohydrate esterases (11.8\%) (Fig. 4c).

We further compared a-diversity in the individuals at the KEGG orthology (KO) level, Cluster of Orthologous Groups (COG) level and family level of CAZy levels (Supplementary Figure S2). There was no significant difference between the chao1 and shannon indies at the KO level and COG level, except that chao1 index at the COG level was significantly different between the XYHXC and QYMC group (Supplementary Figure S2). Moreover, The Shannon index shown that the diversity is similar in all groups 
on the KO and COG levels, and XYHXC group was significantly higher diversity than other groups on the family level of CAZy.

\section{Antibiotic resistance gene (ARG) annotation}

We used the annotation results compare with the CARD, and a total of 989 ARGs were identified in all samples as shown in Table S2. There were some differences in ARGs numbers in the six breeds of broiler. Especially, the number of ARGs in HZXHC and QYMC group were lower than in other groups ((Fig. 5a). Starting from the relative abundance table of AGRs, the ARGs results of the maximum abundance ranking top 20 were shown as histogram (Fig. 5b), and the ARGs abundance in ppm and relative percentage in each broiler was calculated. The results showed that the abundance and percentage of tet(Q) was higher than other ARGs in all samples. Identified ARGs were further categorized on the basis of their resistance profiles, and each ARG was annotated with information of resistance type. The overall presence of these resistance types described as a circos plot (Fig. 6a), which indicated the most predominant types of ARGs in the six groups of chicken are tetracyclines, multidrug and aminoglycoside, and found that the KFXHC group is the highest proportion of resistance type among the six groups. Interestingly, the highest abundance of ARG types in the XYHXC group was also found by comparing the dominant types of ARG in 6 groups of broilers (Fig. 6b), the result analysis implied that Xinghua chicken is more resistant to antibiotics than the other five yellow-feathered broilers.

\section{Discussion}

The yellow-feather broilers are traditionally indigenous chicken, and traditional nutritional and commercial mainstay for millions of Chinese people. Substantial studies highlight the importance of the gut microbiome in broilers' health and performance [14]. Meanwhile, animal intestinal microflora harbors a vast reservoir of ARGs, which are exposed and spread may cause ecological dangers in the environment $[15,16]$. Approximately $85-99 \%$ of intestinal microbes cannot be cultivated in the lab, which limits our understanding of bacteria function including those with antibiotic resistance [17]. The 16s rRNA technology is difficult deciphering functional capabilities of the microbes when focusing only on limited segments of a gene [18]. Therefore, we performed metagenomic method to investigate difference in the gut microbial community structure of indigenous broiler breeds, and their potential to the biosynthesis of antibiotics, antibiotic resistance, the ARGs, and their association with the host microbiome.

The cecal microbiome plays a vital role in the digestion of dietary crude fiber and affects the nutrient digestion and absorption in broilers [19]. Therefore, the cecal microbiome has been extensively studied. In this study, we have performed microbiome metagenome analysis on six breeds indigenous chickens from China. The result found that Bacteroidetes and Firmicutes are the most abundant microbiomes in the six groups of chickens, which was consistent with previous studies [20,21]. Members of these two phyla can produce short-chain fatty acids (SCFAs) in the gut and widely associated with human obesity [22], which play an important role in regulating host energy metabolism [23]. At the genus level, we found that the most dominant genus in indigenous chicken is the Bacteroides, which plays an important role in 
decomposing polysaccharides useful to animal hosts [24]. Furthermore, the major dominant genera of qingyuanPatridge chicken are Prevotella and Bacteroides, and the relative abundance of Prevotella was significantly higher than the other 5 groups. Prevotella abundance has been shown to be important in carbohydrate utilization and can increase host fat accumulation [25,26]. A higher relative abundance of Prevotella was also found in both low and high feed efficiency pigs by Tan et al. [27]. PCoA and NMDS plots showed a clear population structure among samples of the difference lines in yellow-feathered broilers, especially in the Huiyang Bearded chicken, Xinghua chicken, ZhongshanShanlan chicken and qingyuanPatridge chicken. Similar pattern has been reported in previous studies [28]. Several factors may cause variation in the gut microbiota, among them breed, age, and feed [29]. In addition, previous studies revealed the significant influence of host genetics and environmental factors on the composit ion of the gut microbiome [30]. Goodrich et al. have found evidence that the host genome influence on the composition of microbiome in humans [31]. KEGG, eggNOG, and CAZy are the most frequently used databases to study functional properties of the microbial population [32]. Generally, three level classification approaches against all three databases have been used for functional profiling. Metabolism, genetic information processing, cellular processes, human diseases, and organismal systems were the dominant functions predicted at level one in our KEGG pathway analysis.

In the current study, metagenomics sequencing was adopted to identified the presence and relative abundance of ARGs in the yellow-feathered broilers. The study results found that the present of 989 ARGs in the yellow-feathered broilers, including tetQ, adeF, OXA-347, Ermf and tet $(W / N / W)$ et al., and the tetQ gene has the highest relative abundance in all samples. The tet $Q$ gene is a ribosomal protection protein, its gene is associated with a conjugative transposon and has been found in both gram-positive and gram-negative bacteria. We then matched each ARGs to its corresponding antibiotic and concluded the ARGs in the chicken gut microbiota conferred resistance to almost all major antibiotic classes commonly administered for agricultural use. Among them, the tetracycline, multidrug and aminoglycoside contributed to most of the percentage of total resistance in the yellow-feathered chickens. In recent decades, tetracycline has been widely incorporated into animal feed to reduce diseases and increase growth rates, Although the use of antibiotics as growth promoters was banned at 2020 years in China, ARGs had already been deposited on microbial genomes [33], the observation that the broiler gut microbiome has a broad ranging resistome is not surprising. Rovira et al. have found that stop using tetracyclines in farms would not cause an automatic reduction in resistance [34]. Ma et al. have reported that ARGs can transmit from livestock animals to humans and widely spread across different environments [35]. Hence, it is recommended to use antibiotics carefully to prevent the increase and transfer of key ARGs from the farm. Interestingly, we found that the Xinghua chicken have more abundance of resistance genes than other five breeds of the yellow-feathered chickens. Factors influencing the high abundance of ARGs in the Xinghua chicken have not been fully explained. Fodder is a potential source of ARGs in antibiotic resistance of chicken that go through dynamic changes with composition in diet. The farm environment also serves as a potential source of resistant bacteria that can become resident in the chicken gut, but for this complex source it is difficult to explain the drivers of increased resistance in Xinghua chicken gut. Next, PCoA analysis indicated significant differences in the 
structures of ARGs among the six breeds of broilers. We speculated the composition of ARG types would be different because of the distinct microbial community structure and diversity of both feces and the internal environment of the chicken gut [36], or different feeding patterns in the different breeds of broiler [37]. In summary, the results showed that the gut of commercial broilers contained drug-resistant bacteria. Resistant and pathogenic bacteria in feces may cause potential pollution and public health risk, despite great efforts to reduce contamination in the slaughter process. Additional risks may exist due to environmental pollution or to direct exposure of the farm laborer.

\section{Conclusions}

This study demonstrates that the Bacteroidetes and Firmicutes were two dominant phyla, and the Bacteroides were the dominant genus in the yellow-feathered broilers. and found that the relative abundance of Prevotella in the QingyuanPatridge chicken was significantly higher than the other five breeds. The ARGs reported that shown the abundance of antibiotics resistant (tetracyclines, multidrug and aminoglycoside) in each breed, and found Xinghua chicken is more resistant to antibiotics than the other five yellow-feathered broilers. To our knowledge, this study is the first to provide comprehensive microbiomes and antibiotic resistance profiles for Chinese yellow-feathered broilers. The microbial and antibiotic resistant data generated in this study provides a useful indicator of the use of produce in the yellow-feathered broiler.

\section{Abbreviations}

ANOSIM, analysis of similarity; ARG, antibiotic resistance gene; CARD, comprehensive antibiotic resistance databases; CAZy, carbohydrate-active enzymes database; eggNOG, evolutionary genealogy of genes, non-supervised orthologous groups; KEGG, kyoto encyclopedia of genes and genomes; NR, nonredundant protein; OG, orthologous group; ORF, open reading frame; PCoA, principle coordination analysis; NMDS, non-metric multi-dimensional scaling analysis.

\section{Declarations}

\section{Ethics approval and consent to participate}

All animal experiments were performed according to the protocols approved by the South China Agriculture University Institutional Animal Care and Use Committee (approval number SCAU\#2020C029). All animal procedures followed the regulations and guidelines established by this committee and minimized the suffering of animals.

\section{Consent for publication}

Not applicable

\section{Availability of data and material}


All data generated or analyzed during this study are available from the corresponding author on reasonable request.

\section{Competing interests}

The authors declare that they have no competing interests.

\section{Funding}

This work was supported by the Natural Scientific Foundation of China (U1901206 and 31761143014), Local Innovative and Research Teams Project of Guangdong Province (2019BT02N630), Ten-Thousand Talents Program (W03020593), and China Agriculture Research System (CARS-41-G03).

\section{Authors' contributions}

YB participated in the design of the experiment and wrote the manuscript and data analyses. LJ participated in data analyses and engaged in useful discussion and revised the manuscript. YL and SY participated in data collection and data analyses. QN developed the concepts, designed and supervised the study, and revised the manuscript.

\section{Availability of data and materials}

The dataset supporting the conclusions of this article is available in the NCBI short read archive under BioProject number PRJNA764069.

\section{Acknowledgements}

Not applicable.

\section{References}

1. Li L, Abouelezz KFM, Gou Z, Lin X, Wang Y, Fan Q, Cheng Z, Ding F, Jiang S, Jiang Z. Optimization of Dietary Zinc Requirement for Broiler Breeder Hens of Chinese Yellow-Feathered Chicken. Animals (Basel). 2019;9(7). https://doi.org/10.3390/ani9070472

2. Du B, Chen J, Zheng Q, Li W, Zhong F, Zhang X, He D, Zhang J, Huang X. Genetic diversity and population structure of indigenous chicken breeds in South China. Front Agr Sci and Eng. 2016;3(2):97-101. https://doi.org/10.15302/j-fase-2016102

3. Zheng MQ, Gong GF, Gao HJ, Yao WY, Lv SY, Tian LJ, J. W. Report of Chinese broiler industry development in 2015. China Poult. 2016;38:67-70. https://doi.org/10.16372/j.issn.10046364.2016.07.018

4. China National Commission of Animal Genetic Resources. Animal Genetic Resources in China Poultry. Agriculture Press of China; 2011. 
5. Zhu B, Wang X, Li L. Human gut microbiome: the second genome of human body. Protein Cell. 2010;1(8):718-725. https://doi.org/10.1007/s13238-010-0093-z

6. Kohl KD. Diversity and function of the avian gut microbiota. J Comp Physiol B. 2012;182(5):591-602. https://doi.org/10.1007/s00360-012-0645-z

7. Diaz Carrasco JM, Casanova NA, Fernández Miyakawa ME. Microbiota, Gut Health and Chicken Productivity: What Is the Connection? Microorganisms. 2019;7(10).

https://doi.org/10.3390/microorganisms 7100374

8. Stanley D, Geier MS, Denman SE, Haring VR, Crowley TM, Hughes RJ, Moore RJ. Identification of chicken intestinal microbiota correlated with the efficiency of energy extraction from feed. Vet Microbiol. 2013;164(1-2):85-92. https://doi.org/10.1016/j.vetmic.2013.01.030

9. Mancabelli L, Ferrario C, Milani C, Mangifesta M, Turroni F, Duranti S, Lugli GA, Viappiani A, Ossiprandi MC, van Sinderen D et al. Insights into the biodiversity of the gut microbiota of broiler chickens. Environ Microbiol. 2016;18(12):4727-4738. https://doi.org/10.1111/1462-2920.13363

10. Clavijo V, Flórez MJV. The gastrointestinal microbiome and its association with the control of pathogens in broiler chicken production: A review. Poult Sci. 2018;97(3):1006-1021. https://doi.org/10.3382/ps/pex359

11. Oakley BB, Lillehoj HS, Kogut MH, Kim WK, Maurer JJ, Pedroso A, Lee MD, Collett SR, Johnson TJ, Cox NA. The chicken gastrointestinal microbiome. FEMS Microbiol Lett. 2014;360(2):100-112. https://doi.org/10.1111/1574-6968.12608

12. Van Boeckel TP, Brower C, Gilbert M, Grenfell BT, Levin SA, Robinson TP, Teillant A, Laxminarayan R. Global trends in antimicrobial use in food animals. Proc Natl Acad Sci U S A. 2015;112(18):56495654. https://doi.org/10.1073/pnas.1503141112

13. Qiao M, Ying GG, Singer AC, Zhu YG. Review of antibiotic resistance in China and its environment. Environ Int. 2018;110:160-172. https://doi.org/10.1016/j.envint.2017.10.016

14. Ji J, Xu Y, Luo C, He Y, Xu X, Yan X, Li Y, Shu D, Qu H. Effects of the DMRT1 genotype on the body weight and gut microbiota in the broiler chicken. Poult Sci. 2020;99(8):4044-4051. https://doi.org/10.1016/j.psj.2020.03.055

15. Xiong W, Wang Y, Sun Y, Ma L, Zeng Q, Jiang X, Li A, Zeng Z, Zhang T. Antibiotic-mediated changes in the fecal microbiome of broiler chickens define the incidence of antibiotic resistance genes. Microbiome. 2018;6(1):34. https://doi.org/10.1186/s40168-018-0419-2

16. Kang K, Hu Y, Wu S, Shi S. Comparative Metagenomic Analysis of Chicken Gut Microbial Community, Function, and Resistome to Evaluate Noninvasive and Cecal Sampling Resources. Animals (Basel). 2021;11(6). https://doi.org/10.3390/ani11061718

17. Lok C. Mining the microbial dark matter. Nature. 2015;522(7556):270-273. https://doi.org/10.1038/522270a

18. Kröber M, Bekel T, Diaz NN, Goesmann A, Jaenicke S, Krause L, Miller D, Runte KJ, Viehöver P, Pühler $A$ et al. Phylogenetic characterization of a biogas plant microbial community integrating clone library 
16S-rDNA sequences and metagenome sequence data obtained by 454-pyrosequencing. $J$ Biotechnol. 2009;142(1):38-49. https://doi.org/10.1016/j.jbiotec.2009.02.010

19. Rodrigues DR, Briggs W, Duff A, Chasser K, Murugesan R, Pender C, Ramirez S, Valenzuela L, Bielke L. Cecal microbiome composition and metabolic function in probiotic treated broilers. PLoS One. 2020;15(6):e0225921. https://doi.org/10.1371/journal.pone.0225921

20. Kwak MJ, Park MY, Choi YS, Cho J, Pathiraja D, Kim J, Lee H, Choi IG, Whang KY. Dietary sophorolipid accelerates growth by modulation of gut microbiota population and intestinal environments in broiler chickens. J Anim Sci Biotechnol. 2021;12(1):81. https://doi.org/10.1186/s40104-021-00606-x

21. Maki JJ, Bobeck EA, Sylte MJ, Looft T. Eggshell and environmental bacteria contribute to the intestinal microbiota of growing chickens. J Anim Sci Biotechnol. 2020;11:60.

https://doi.org/10.1186/s40104-020-00459-w

22. Mariat D, Firmesse O, Levenez F, Guimarăes V, Sokol H, Doré J, Corthier G, Furet JP. The Firmicutes/Bacteroidetes ratio of the human microbiota changes with age. BMC Microbiol. 2009;9:123. https://doi.org/10.1186/1471-2180-9-123

23. Murugesan S, Ulloa-Martínez M, Martínez-Rojano H, Galván-Rodríguez FM, Miranda-Brito $C$, Romano MC, Piña-Escobedo A, Pizano-Zárate ML, Hoyo-Vadillo C, García-Mena J. Study of the diversity and short-chain fatty acids production by the bacterial community in overweight and obese Mexican children. Eur J Clin Microbiol Infect Dis. 2015;34(7):1337-1346. https://doi.org/10.1007/s10096-0152355-4

24. Huang Y, Lv H, Song Y, Sun C, Zhang Z, Chen S. Community composition of cecal microbiota in commercial yellow broilers with high and low feed efficiencies. Poult Sci. 2021;100(4):100996. https://doi.org/10.1016/j.psj.2021.01.019

25. Chen C, Fang S, Wei H, He M, Fu H, Xiong X, Zhou Y, Wu J, Gao J, Yang H et al. Prevotella copri increases fat accumulation in pigs fed with formula diets. Microbiome. 2021;9(1):175. https://doi.org/10.1186/s40168-021-01110-0

26. Han X, Yang Y, Yan H, Wang X, Qu L, Chen Y. Rumen bacterial diversity of 80 to 110-day-old goats using 16S rRNA sequencing. PLoS One. 2015;10(2):e0117811. https://doi.org/10.1371/journal.pone.0117811

27. Tan Z, Yang T, Wang Y, Xing K, Zhang F, Zhao X, Ao H, Chen S, Liu J, Wang C. Metagenomic Analysis of Cecal Microbiome Identified Microbiota and Functional Capacities Associated with Feed Efficiency in Landrace Finishing Pigs. Front Microbiol. 2017;8:1546. https://doi.org/10.3389/fmicb.2017.01546

28. Miller EA, Winfield B, Weber BP, Flores-Figueroa C, Munoz-Aguayo J, Huisinga J, Johnson TJ. Convergence of the turkey gut microbiota following cohabitation under commercial settings. J Anim Sci Biotechnol. 2021;12(1):59. https://doi.org/10.1186/s40104-021-00580-4

29. Wang X, Tsai T, Deng F, Wei X, Chai J, Knapp J, Apple J, Maxwell CV, Lee JA, Li Y et al. Longitudinal investigation of the swine gut microbiome from birth to market reveals stage and growth performance associated bacteria. Microbiome. 2019;7(1):109. https://doi.org/10.1186/s40168-019$0721-7$ 
30. Crespo-Piazuelo D, Migura-Garcia L, Estellé J, Criado-Mesas L, Revilla M, Castelló A, Muñoz M, García-Casco JM, Fernández Al, Ballester M et al. Association between the pig genome and its gut microbiota composition. Sci Rep. 2019;9(1):8791. https://doi.org/10.1038/s41598-019-45066-6

31. Goodrich JK, Davenport ER, Clark AG, Ley RE. The Relationship Between the Human Genome and Microbiome Comes into View. Annu Rev Genet. 2017;51:413-433. https://doi.org/10.1146/annurevgenet-110711-155532

32. Kumar H, Park W, Lim D, Srikanth K, Kim JM, Jia XZ, Han JL, Hanotte O, Park JE, Oyola SO. Whole metagenome sequencing of cecum microbiomes in Ethiopian indigenous chickens from two different altitudes reveals antibiotic resistance genes. Genomics. 2020;112(2):1988-1999. https://doi.org/10.1016/j.ygeno.2019.11.011

33. Segura-Wang M, Grabner N, Koestelbauer A, Klose V, Ghanbari M. Genome-Resolved Metagenomics of the Chicken Gut Microbiome. Front Microbiol. 2021;12:726923. https://doi.org/10.3389/fmicb.2021.726923

34. Rovira P, McAllister T, Lakin SM, Cook SR, Doster E, Noyes NR, Weinroth MD, Yang X, Parker JK, Boucher $\mathrm{C}$ et al. Characterization of the Microbial Resistome in Conventional and "Raised Without Antibiotics" Beef and Dairy Production Systems. Front Microbiol. 2019;10:1980. https://doi.org/10.3389/fmicb.2019.01980

35. Ma L, Xia Y, Li B, Yang Y, Li LG, Tiedje JM, Zhang T. Metagenomic Assembly Reveals Hosts of Antibiotic Resistance Genes and the Shared Resistome in Pig, Chicken, and Human Feces. Environ Sci Technol. 2016;50(1):420-427. https://doi.org/10.1021/acs.est.5b03522

36. Huang P, Zhang Y, Xiao K, Jiang F, Wang H, Tang D, Liu D, Liu B, Liu Y, He X et al. The chicken gut metagenome and the modulatory effects of plant-derived benzylisoquinoline alkaloids. Microbiome. 2018;6(1):211. https://doi.org/10.1186/s40168-018-0590-5

37. Jochum JM, Redweik GAJ, Ott LC, Mellata M. Bacteria Broadly-Resistant to Last Resort Antibiotics Detected in Commercial Chicken Farms. Microorganisms. 2021;9(1). https://doi.org/10.3390/microorganisms9010141

\section{Figures}


a

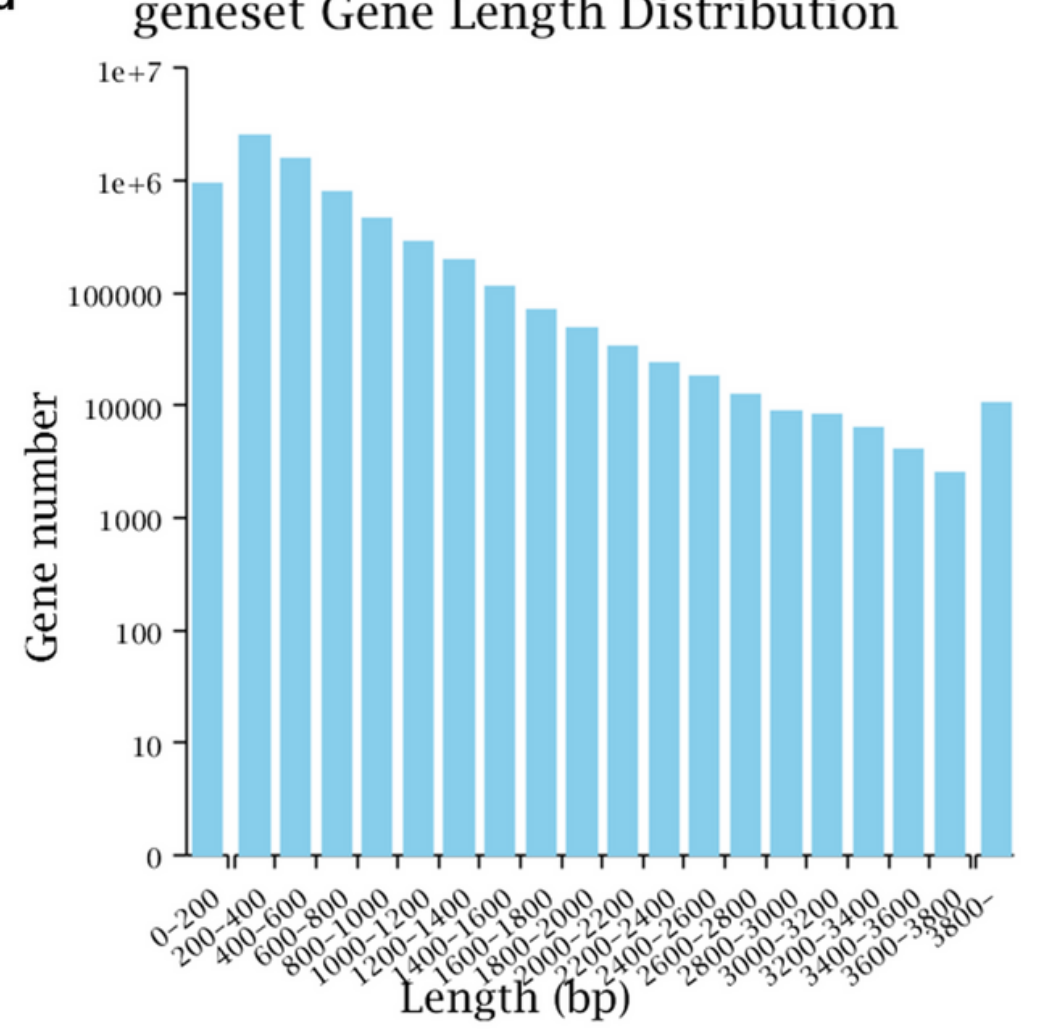

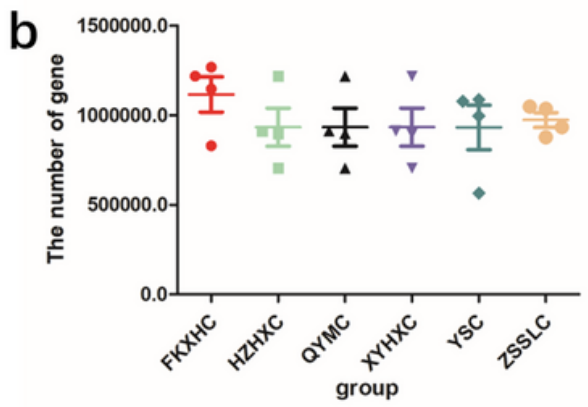

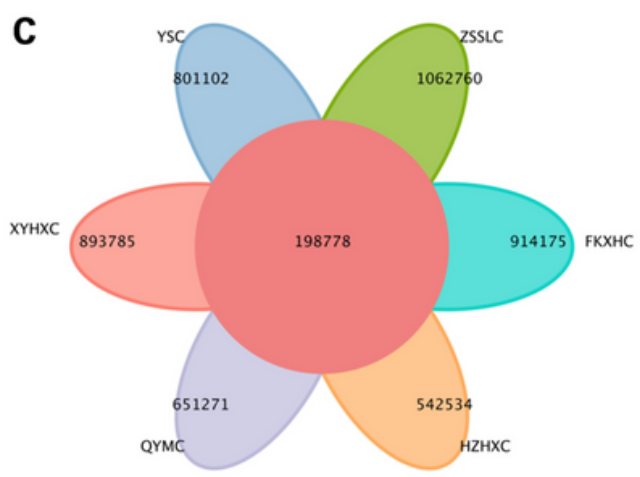

\section{Figure 1}

Gene abundance statistics. a Gene length distribution, X-axis: length of the genes, Y-axis: number of genes of the total genes. $b$ Scatter dot plot showing the difference in gene numbers among the six groups. $c$ Venn diagram showing the numbers of common and unique genes among the six groups. Data are expressed as means \pm SEM, ${ }^{\star} p<0.05 ;{ }^{* \star} p<0.01 ; * \star * p<0.001$. 

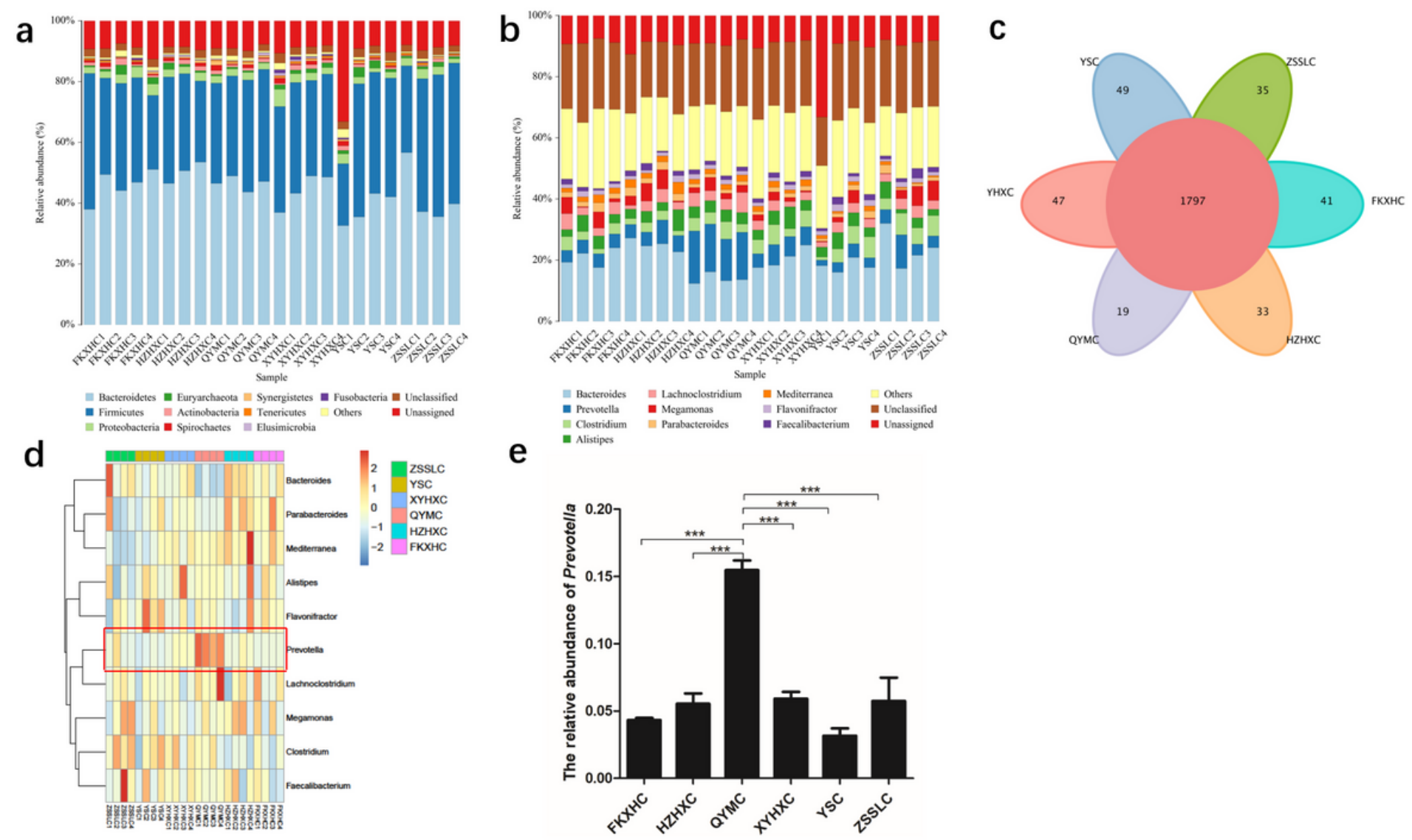

\section{Figure 2}

Taxonomic annotation. a Relative abundance at phylum level. b Relative abundance at genus level. c Venn diagram showing the number of common and unique genus among the six groups. $d$ Heatmap representation of taxonomy abundance of the Top 10 dominant genera. e Histogram showing the difference in the relative abundance of Prevotella among the six groups. Data are expressed as means \pm SEM, ${ }^{*} p<0.05 ; * * p<0.01 ; * \star * p<0.001$. 

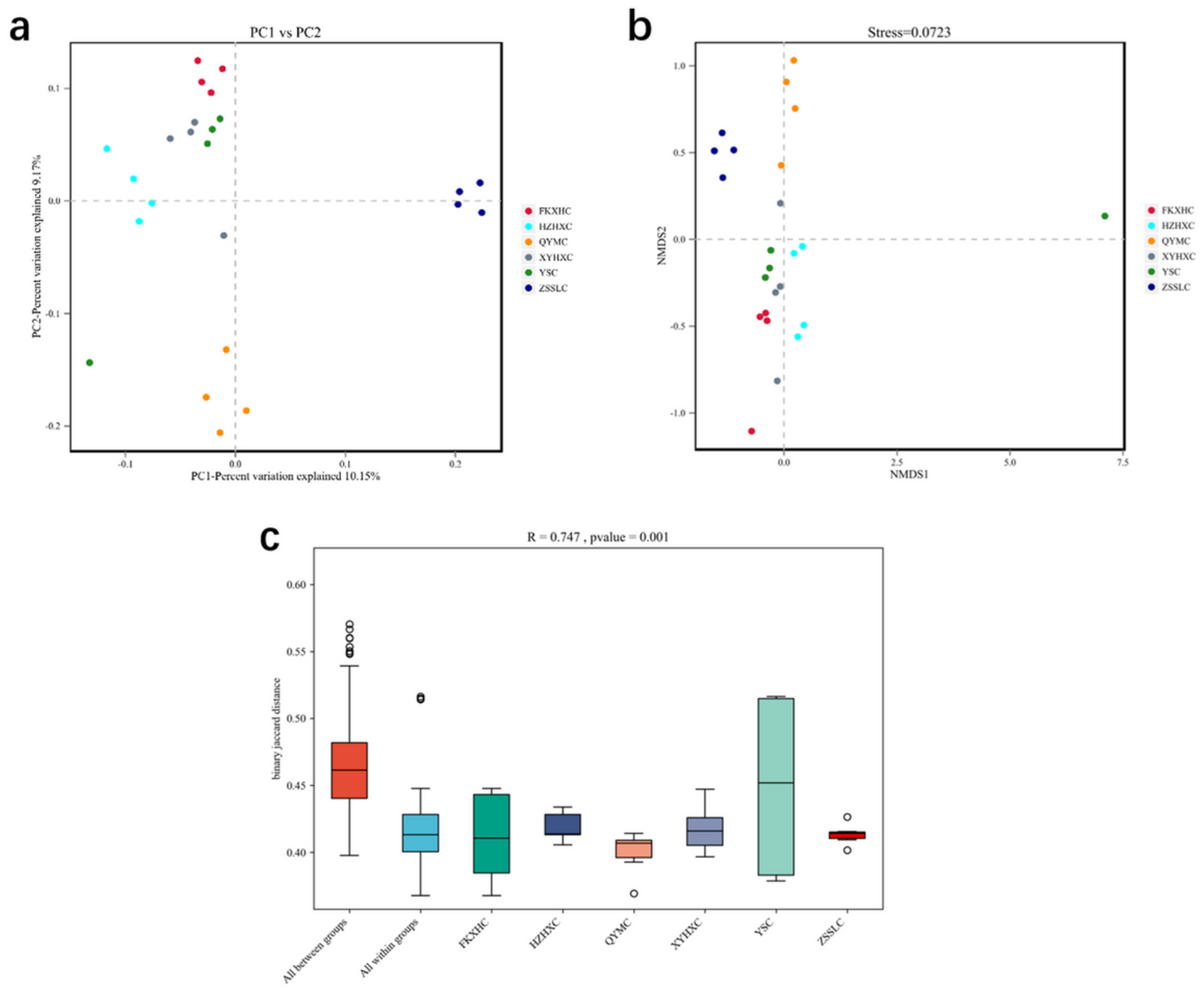

Figure 3

beta-diversity analysis. a PCoA plot and b NMDS plot of binary-jaccard distances among six groups. c ANOSIM plots for variations among six groups. 
a

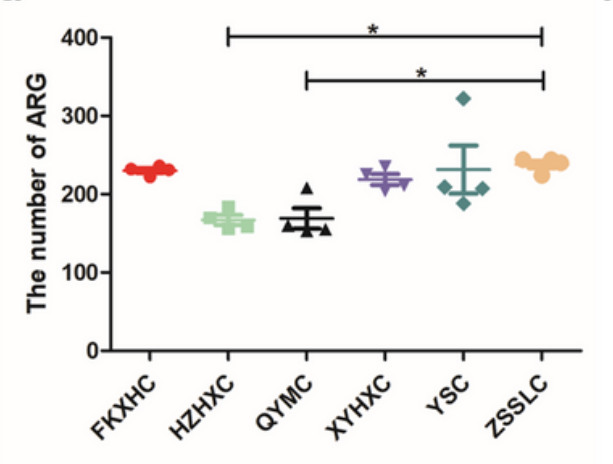

C b

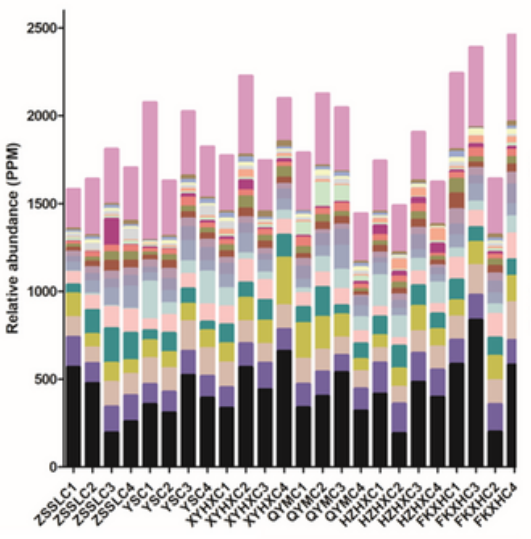

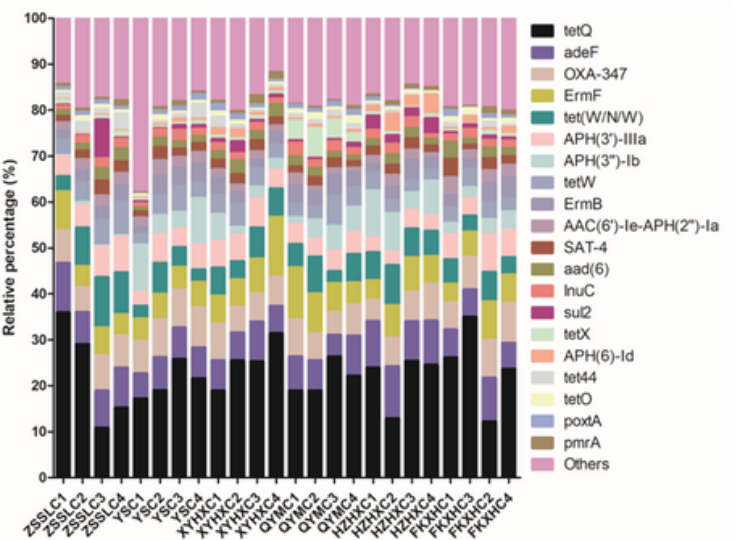

$R=0.525$, pvalue $=0.001$
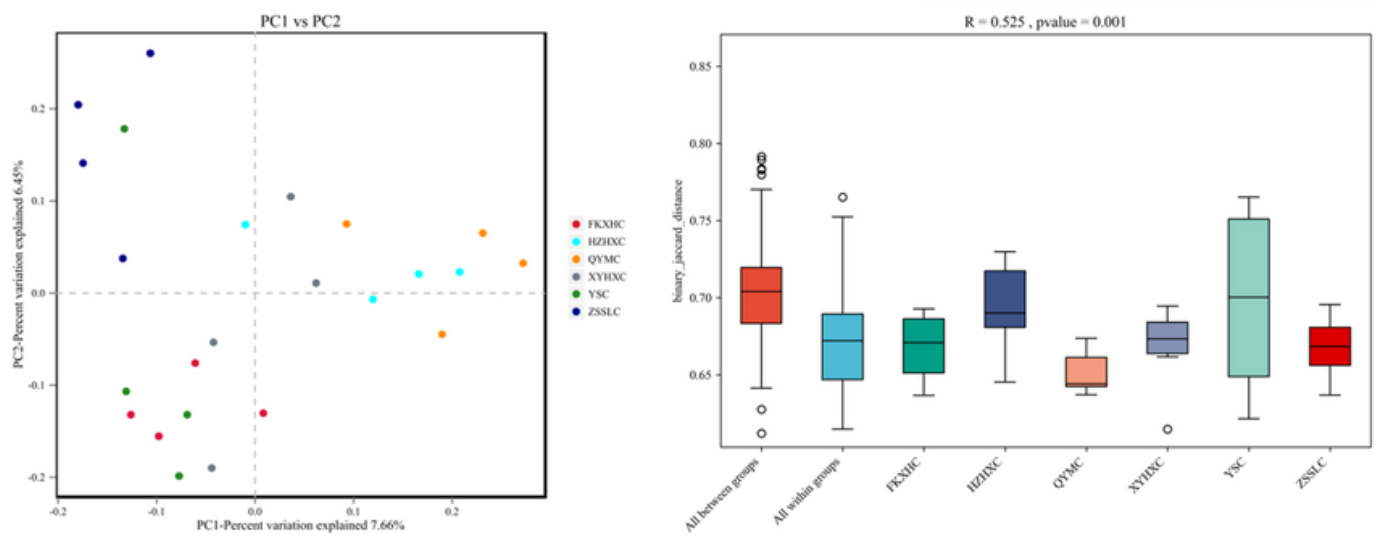

\section{Figure 4}

Functional annotation. a Statistical map of KEGG metabolic pathway-related functional genes at the level $2, \mathrm{X}$-axis: the relative content of the corresponding functional gene number, $\mathrm{Y}$-axis: the classification content of KEGG leve1 and leve2. b Histogram of eggNOG functional gene function classification, $X$-axis: the relative content of the corresponding functional gene number, $Y$-axis: the classification content of eggNOG. c Pie chart of the proportion of various carbohydrate enzyme. 
a

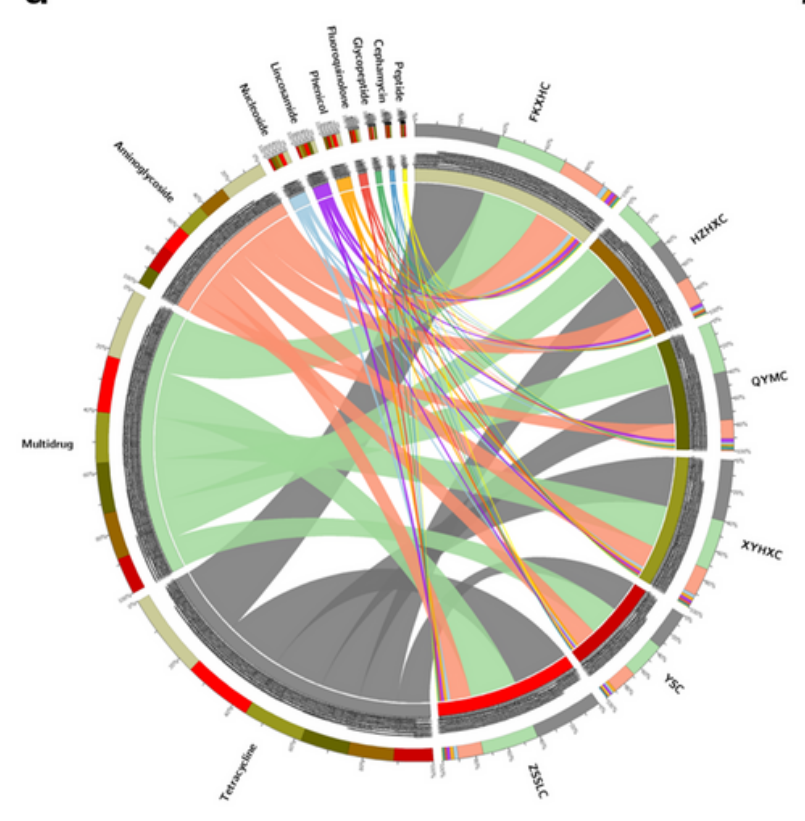

b
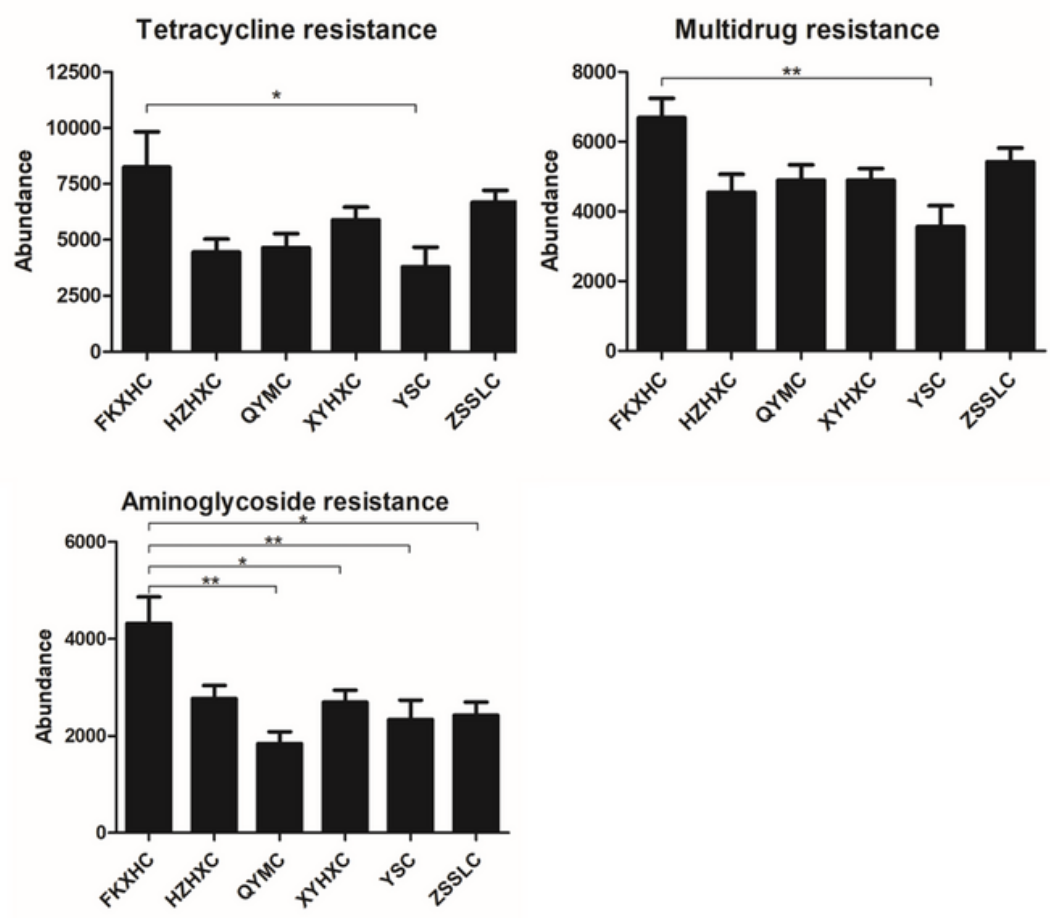

Figure 5

The abundance of antibiotic resistant gene. a Scatter dot plot showing the difference in the number of ARGs among the six groups. $b$ The relative abundance in unit PPM of magnifying 106 times of original abundance and the percentage of ARGs in each sample. c PCoA plot of binary-jaccard distances among six groups. d ANOSIM plots for variations among six groups.

\section{Image not available with this version}

\section{Figure 6}

The abundance of antibiotic resistant types. a Circos plot of relative abundance of ARG types, right side of the circle indicates group information, left side indicates antibiotic information, outer circle is the ideogram scale of distribution of unique genes, and inner circle represents different groups or antibiotics.streptomycin biosynthesis. b Histogram showing the difference abundance in the resistance genus among the six groups. Data are expressed as means $\pm \mathrm{SEM},{ }^{\star} \mathrm{p}<0.05 ;{ }^{\star \star} \mathrm{p}<0.01 ;{ }^{\star \star \star} \mathrm{p}<0.001$.

\section{Supplementary Files}

This is a list of supplementary files associated with this preprint. Click to download. 
- supplementalfigures.docx

- supplementaltables.xlsx 